\title{
ESCORRENTÍA Y PÉRDIDA DE SUELO EN CÁRCAVAS: MODELIZACIÓN EN UN AGROSISTEMA DEL PREPIRINEO OSCENSE
}

\author{
M. LÓPEZ-VICENTE y A. NAVAS \\ Departamento de Suelo y Agua \\ Estación Experimental de Aula Dei - CSIC, Apartado 13034, 50080 Zaragoza \\ correo electrónico de contacto: mvicente@eead.csic.es
}

RESUMEN. La dinámica erosiva de las cárcavas en ambientes mediterráneos constituye una de las principales causas de degradación del suelo de los agrosistemas productivos y la principal vía de exportación de partículas erosionadas. En este trabajo se aplica el modelo semifísico RMMF corregido para la modelización espacialmente distribuida en un SIG de la escorrentía acumulada potencial y efectiva y de la erosión del suelo en la cuenca de la Cárcava La Coloma, situada en el Prepirineo Central oscense. La cárcava alcanza una longitud máxima de 523 m y está dividida en cuatro sectores atendiendo a sus características fisiográficas. El mapa de desagregación del suelo por salpicadura refleja la importancia de la cubierta vegetal en las tasas de erosión obtenidas, mientras que los procesos de acumulación de la escorrentía, de infiltración y de pendiente explican los valores estimados de erosión en el mapa de desagregación por escorrentía. En el conjunto de la cuenca la capacidad de transporte de la escorrentía es el factor limitante de la erosión en el $88 \%$ de la superficie, la erosión promedio es $33.9 \mathrm{Mg} \mathrm{ha}^{-1}$ año-1 y la pérdida total de suelo suma $411.4 \mathrm{Mg}^{-1} \tilde{n} o^{-}$ 1. La erosión promedio estimada en la cárcava es de $176.5 \mathrm{Mg} \mathrm{ha}^{-1}$ año-1 con valores que oscilan entre 0.1 y $502.6 \mathrm{Mg} \mathrm{ha}^{-1}$ año-1 para los distintos sectores identificados y el volumen total de escorrentía generada es de $779 \mathrm{~mm}^{2}$ ño ${ }^{-1}$. Los campos de cultivo, caminos, zonas de bosque mediterráneo abierto y arbustos dispersos y las áreas de fuerte pendiente son las principales fuentes de suelo erosionado al canal principal de la cárcava, con tasas de erosión superiores a 30 $\mathrm{Mg} \mathrm{ha^{-1 }}$ año ${ }^{-1}$. La información generada mediante el modelo RMMF corregido puede servir para implementar medidas de conservación del suelo y del agua para el desarrollo sostenible de la cuenca de estudio y reducir la dinámica erosiva de la Cárcava La Coloma.

ABSTRACT. Erosive dynamic of gullies in Mediterranean environments represents one the main causes of land degradation and sediment yield in productive 
agrosystems. In this work the semi-physics enhanced RMMF model is applied into a GIS to elaborate spatially distributed maps of potential and effective cumulative runoff and soil erosion at the La Coloma Gully catchment (Spanish Central Pre-Pyrenees). The gully reaches a maximum length of $523 \mathrm{~m}$ and is divided into four different sectors according to its physiographic properties. The map of soil detachment by splash highlights the key role of vegetation properties to explain the different estimated rates. Processes of runoff accumulation and infiltration and slope values mainly control the estimated values of soil detachment by runoff. The average value of soil erosion at catchment scale is $33.9 \mathrm{Mg} \mathrm{ha}^{-1} \mathrm{yr}^{-1}$ being runoff transport capacity the limiting factor in $88 \%$ of the surface and total soil loss adds up to $411.4 \mathrm{Mg} \mathrm{rr}^{-1}$. The average value of estimated soil erosion in the gully is $176.5 \mathrm{Mg} \mathrm{ha}^{-1} \mathrm{yr}^{-1}$ with values that range between 0.1 and $502.6 \mathrm{Mg} \mathrm{ha}^{-1} \mathrm{yr}^{-1}$ for the different sectors and the total volume of runoff is $779 \mathrm{~mm} \mathrm{yr}^{-1}$. Crops, paths and areas with low vegetation cover and high slope are the main source areas of sediment to the main channel of the gully and present high values of soil erosion (higher than $30 \mathrm{Mg} \mathrm{ha}^{-1} \mathrm{yr}^{-1}$ ). Information gained with the enhanced RMMF model is of interest to implement soil and water conservation practices and develop sustainable economic and environmental strategies.

Palabras clave: cárcava, escorrentía, erosión de suelo, SIG, modelo RMMF, algoritmo de flujo acumulado, Prepirineo central.

Key words: gully, runoff, soil erosion, GIS, RMMF model, flow accumulation algorithm, Spanish Central Pre-Pyrenees.

Enviado el 15 de septiembre de 2008 Aceptado el 16 de octubre de 2008

\section{Introducción}

La aparición de sistemas de cárcavas y de paisajes tipo badland se debe a la conjunción de eventos de lluvia torrencial sobre zonas con poca o nula cubierta de vegetación y sobre un determinado tipo de suelo y roca madre. La tipología del substrato y del movimiento del agua superficial y subsuperficial condicionan en gran medida la aparición de las cárcavas siendo frecuente su desarrollo sobre materiales arcillosos, limosos, regolitos, y arenas y conglomerados poco consolidados. No obstante, las actividades agrícolas y la deforestación asociada son en muchos casos el factor desencadenante de la erosión en canales de incisión que dan lugar a la aparición de cárcavas (MenéndezDuarte et al., 2007).

La investigación de los procesos de erosión, transporte y exportación del suelo y sedimento en cárcavas se realiza generalmente a través de medidas directas en parcelas y cuencas monitorizadas (Castillo et al., 2007; Desir y Marín, 2007) o indirectas mediante el análisis de cambios en la topografía (Ries y Marzolff, 2003) y en menor 
medida mediante modelización (e.g. Gordon et al., 2007; Bou Kheir et al., 2008). La escasez de estudios de modelización de la escorrentía y pérdida de suelo en cárcavas se debe, principalmente, a las limitaciones de los modelos aplicados, que en su mayoría representan procesos de erosión por salpicadura, laminar, en regueros y cárcavas efímeras, sin atender a la erosión que se produce en canales, cárcavas permanentes, "piping" o movimientos en masa (de Vente et al., 2008).

En España, el estudio de la dinámica erosiva de las cárcavas se ha centrado tradicionalmente en las regiones de clima semiárido, como en Murcia, en Almería (Faulkner et al., 2004), en la zona centro de Navarra (De Santisteban et al., 2006), en las Bardenas o en la Depresión Central del Ebro (Marzolff y Ries, 2007). En estas regiones semiáridas la actividad de las cárcavas está limitada en el tiempo a la aparición de eventos de lluvia erosiva que ocurren unas pocas veces al año. En menor medida y de modo más reciente aparecen trabajos sobre el desarrollo y evolución de las cárcavas en zonas subhúmedas y húmedas peninsulares, como las que tienen lugar sobre las formaciones margosas y arenosas del flysch eoceno del Pirineo oscense (Nadal-Romero et al., 2008), sobre los regolitos de la Cordillera Cantábrica (Menéndez-Duarte et al., 2007), los conglomerados poco consolidados del sector septentrional de la Cuenca Terciaria del Duero (Fernández et al., 2008) o sobre las margas de la Depresión Terciaria del Penedés (Martínez-Casasnovas et al., 2003).

La elaboración de mapas precisos de circulación de la escorrentía y de su área contributiva unitaria es fundamental para la comprensión de la redistribución espacial del agua y del sedimento a escala de parcela y de cuenca, así como de interés en la identificación de las zonas preferentes de erosión linear, tanto en las divisorias como a lo largo de la ladera (López-Vicente et al., 2006; Chaplot et al., 2005). La localización de las áreas expuestas a altas tasas de erosión es uno de los asuntos de mayor importancia para el desarrollo de planes de conservación del suelo y de gestión del agua. Además, el valor socio-económico por hectárea de cultivo se ha incrementado en los últimos años, haciendo aún más necesaria la prevención de la pérdida de suelo en agrosistemas productivos.

El objetivo de este trabajo es modelizar en detalle y estimar la escorrentía superficial acumulada y la erosión del suelo que tienen lugar en una pequeña cuenca de las Sierras Exteriores del Pirineo oscense asociada a una cárcava desarrollada en un clima subhúmedo. Para lograr este objetivo se aplica el modelo de procesos RMMF (Revised Morgan, Morgan and Finney model; Morgan, 2001) corregido según López-Vicente et al. (2008a) para considerar procesos espacialmente distribuidos de escorrentía potencial y efectiva mediante sistemas de información geográfica (SIG). Los mapas de escorrentía efectiva y erosión de suelo permitirán identificar las principales áreas susceptibles de sufrir procesos severos de degradación del suelo, así como las principales fuentes de sedimento a la cárcava. Estos resultados servirán para orientar futuras medidas de control y prevención de la erosión que ayuden a preservar los recursos hídricos y edáficos hacia el desarrollo sostenible de la zona. 


\section{Material y métodos}

\section{1. Área de estudio}

La cuenca de drenaje de la Cárcava La Coloma (12.2 ha) se sitúa a una altitud de entre 706 y 843 m s.n.m., en la parte central de las Sierras Exteriores pirenaicas (LópezVicente et al., 2009) y dentro de una sub-cuenca de la Cuenca lacustre de Estaña, con una pendiente media del 27.4\%. La Cárcava La Coloma ocupa una extensión de 0.8 ha, se desarrolla de modo continuo, sin interrupciones y tiene una longitud y profundidad máximas de 523 y 1.5 m, respectivamente, con una pendiente media del 32.8\% (Fig. 1). La cuenca de la Cárcava La Coloma drena al lago conocido como "Estanque de Arriba", situado a unos $300 \mathrm{~m}$ al este del pie de la cárcava.

Las Sierras Exteriores constituyen un brusco contraste tectónico entre la Depresión del Ebro y la zona sur-pirenaica, estructuradas en cabalgamientos de gran desarrollo de dirección ONO-ESE y de gran valor morfológico debido al marcado contraste litológico entre estas unidades. Este relieve actúa, además, de barrera climática entre las zonas semiáridas al sur y sub-húmedas y húmedas al norte. La cuenca de estudio se sitúa sobre materiales Mesozoicos y Neógenos, compuestos por dolomías y calizas, margas yesíferas con ocasionales afloramientos masivos de yeso y depósitos coluviales de ladera y de relleno de fondo de valle plano (Fig. 2). El paisaje de esta zona está controlado en parte por procesos kársticos que afectan a los depósitos evaporíticos y carbonatados, dando lugar al desarrollo de dolinas y lagos alimentados principalmente por aguas subterráneas.

La cabecera de la Cárcava La Coloma se sitúa muy próxima a la divisoria de la cuenca y a lo largo de su cauce se observa una ausencia casi total de vegetación, por lo que el retroceso aguas arriba de la pared inicial de la cárcava se considera como un proceso activo en la actualidad. La parte final de la cárcava se sitúa sobre los materiales de relleno de fondo de valle plano, en una zona de muy baja pendiente. El depósito coluvial de ladera aparece afectado por procesos de erosión, y la localización espacial del depósito sugiere una época precedente de acumulación. A lo largo de la cárcava de estudio no se han observado procesos relevantes de "piping" ni de "sapping", ya que la cárcava se desarrolla en su totalidad sobre Facies Keüper, sin intercalación de materiales de diferente comportamiento hidrológico.

Los usos del suelo de la cuenca de estudio son característicos de los agrosistemas mediterráneos en los que se produce una fuerte compartimentación del paisaje. El 9.2\% de la superficie son cultivos de cereal de invierno de secano, el 9.3\% campos abandonados y pastos, el $80.6 \%$ corresponde a diferentes tipos de cubiertas de vegetación natural y el $0.9 \%$ restante a caminos (Fig. 3).

El clima de la zona de estudio es de tipo mediterráneo continental, con un periodo estival seco y dos periodos húmedos en primavera y otoño, y con frecuentes eventos de alta intensidad durante los meses de verano que dan lugar a las mayores tasas de pérdi- 
da de suelo durante el año (López-Vicente et al., 2008b). La precipitación promedio anual es de $595 \mathrm{~mm}$ para el periodo $1993-2006$, con una intensidad máxima $\left(I_{30}\right)$ promedio de $15.1 \mathrm{~mm} \mathrm{~h}^{-1}$ y un valor de lluvia media por día de lluvia erosiva de $35.9 \mathrm{~mm}$ día ${ }^{-1}$ (Fig. 1).

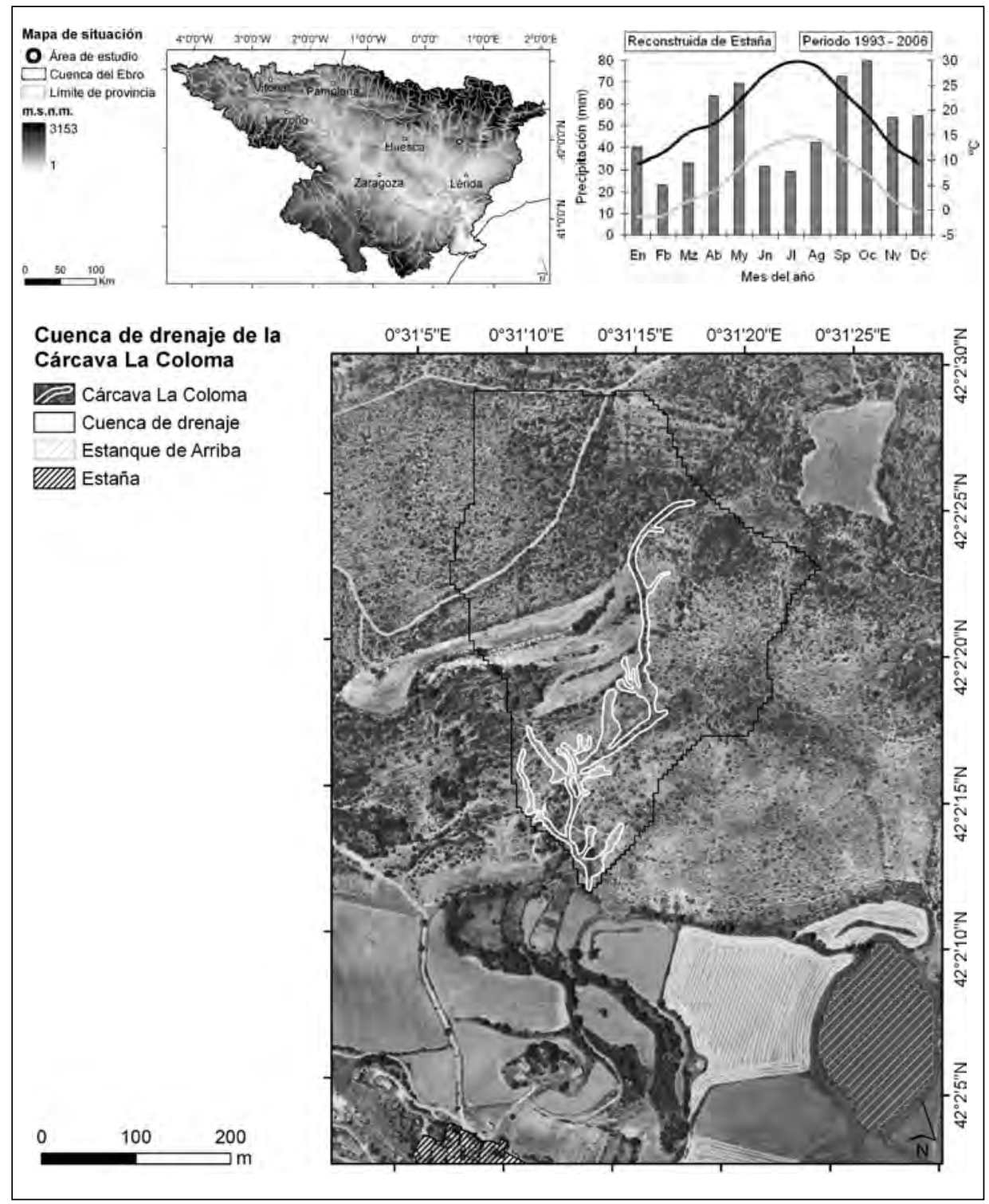

Figura 1. Situación geográfica de la zona de estudio en la provincia de Huesca (a).

Precipitación promedio mensual en la estación climática reconstruida de Estaña para el periodo 1993-2006 (b). Imagen aérea del contorno y cuenca de drenaje de la Cárcava La Coloma (c) 


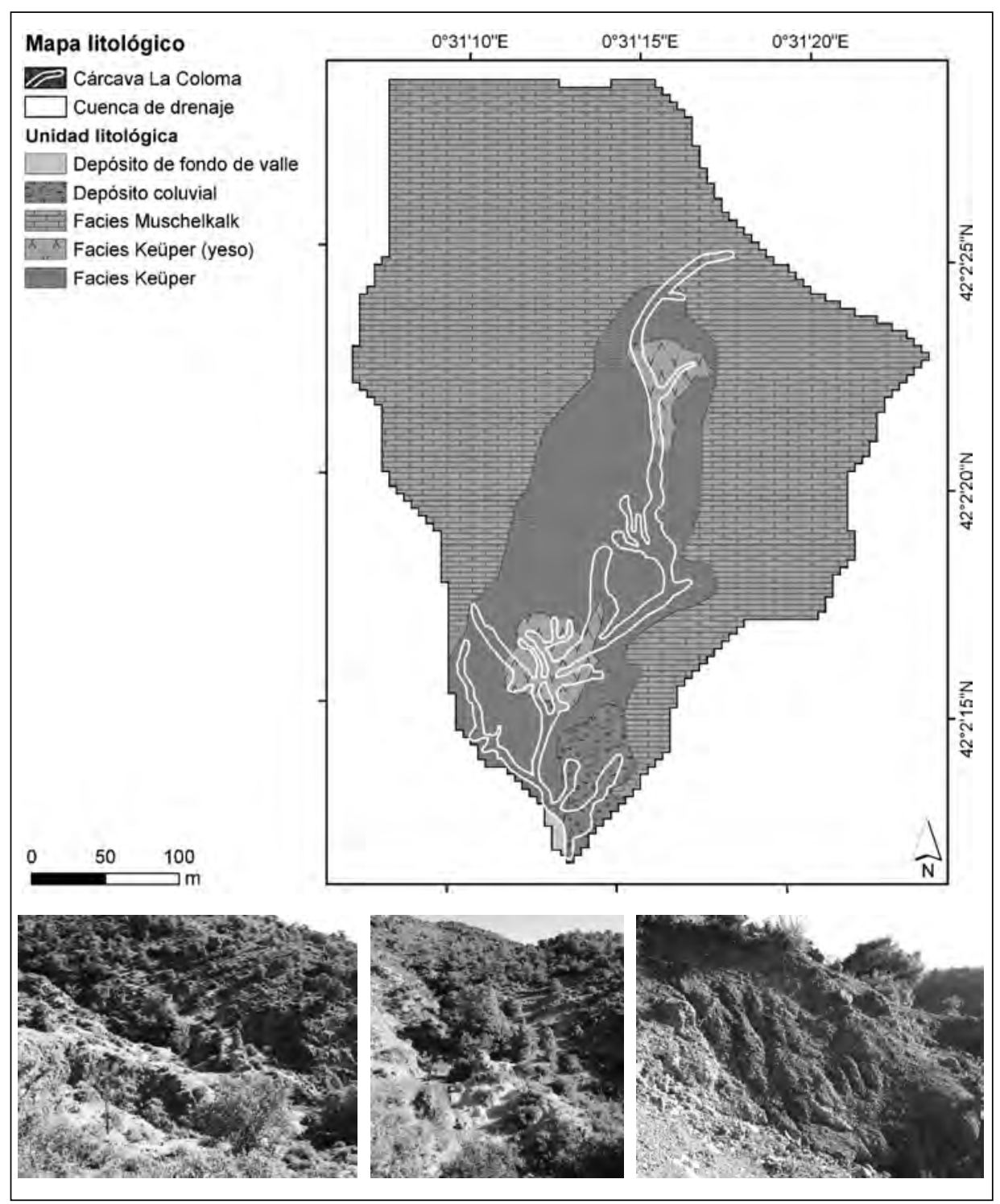

Figura 2. Mapa litológico de la cuenca de la Cárcava La Coloma (Cuenca de Estaña, Huesca)

(a). Ilustración de la cárcava desarrollada sobre materiales arcillo- yesíferos de la Facies Keüper por erosión hídrica de flujo múltiple y sencillo, e imagen del depósito coluvial erosionado (b) 


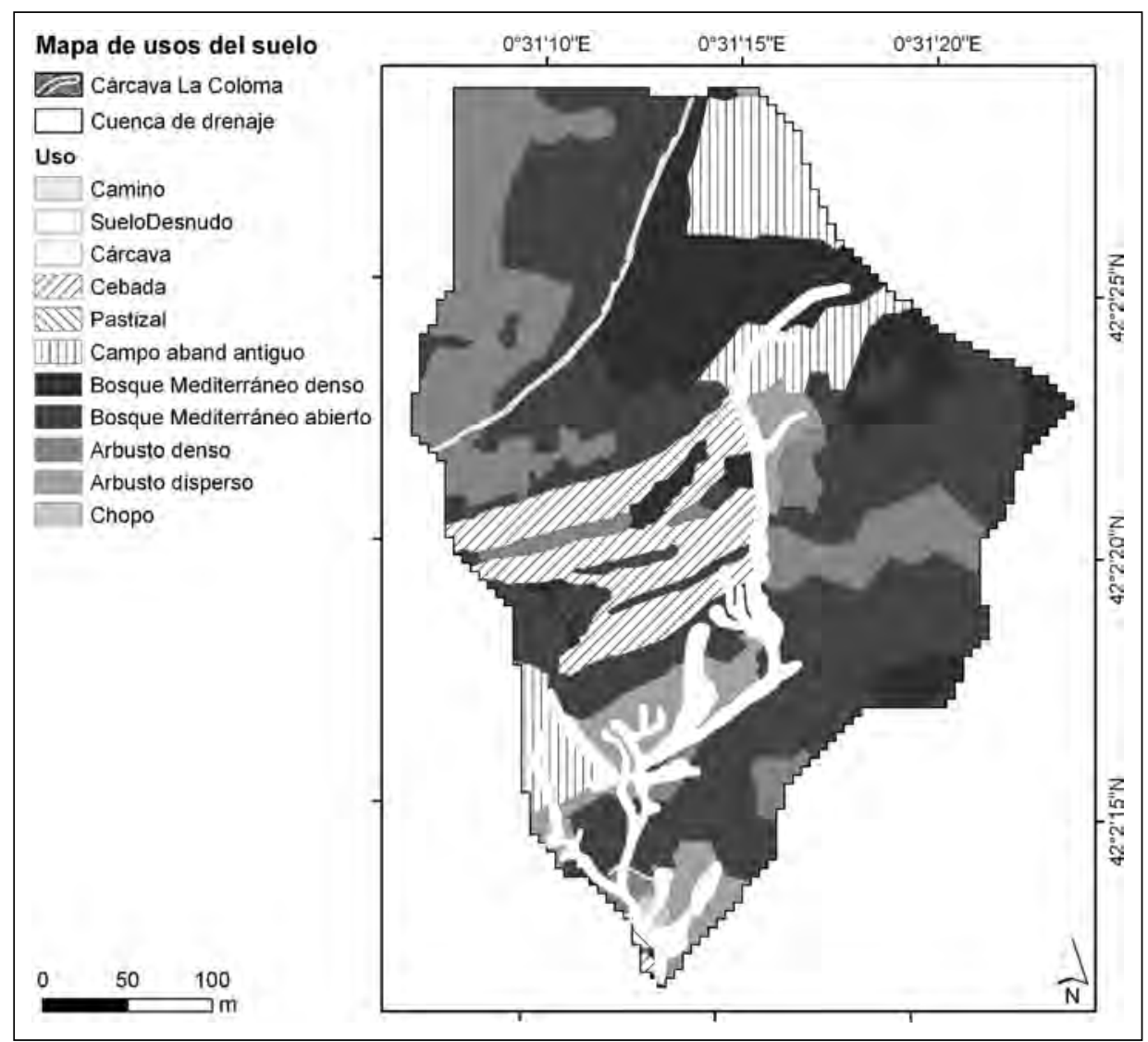

Figura 3. Mapa de usos del suelo de la cuenca de la Cárcava La Coloma.

\subsection{El modelo RMMF corregido de pérdida de suelo}

El modelo RMMF (Revised Morgan, Morgan and Finney model; Morgan, 2001) de base física predice la pérdida promedio anual de suelo a partir de la capacidad de transporte de la escorrentía y de las tasas de desagregación del suelo por salpicadura y por escorrentía. El modelo RMMF es la versión ampliada del modelo MMF (Morgan, Morgan and Finney model; Morgan et al., 1984) de pérdida de suelo, y ha sido aplicado en cuencas y parcelas de numerosos países y ambientes climáticos (e.g. Morgan, 2001; Vigiak et al., 2006). Este modelo no considera el transporte o desplazamiento de partículas del suelo por salpicadura, ni el efecto de las costras superficiales en los procesos de infiltración, ni procesos de erosión en cursos de agua permanente. El modelo RMMF tampoco considera procesos de transporte, exportación y acumulación del suelo erosionado. 
En este trabajo se ha aplicado el modelo RMMF corregido según López-Vicente et al. (2008a) para el cálculo de la escorrentía efectiva mediante un algoritmo de flujo acumulado combinado asociado al inicio de las cárcavas y de factores de infiltración y de microtopografía del suelo (Fig. 4). La pérdida anual de suelo ( $\left.E, \mathrm{Mg} \mathrm{ha}^{-1} \mathrm{año}^{-1}\right)$ se calcula matemáticamente como el valor mínimo entre la suma de las tasas de desagregación del suelo por salpicadura $\left(F, \mathrm{Mg} \mathrm{ha}^{-1} \mathrm{año}^{-1}\right)$ y por escorrentía $\left(H, \mathrm{Mg} \mathrm{ha}^{-1} \mathrm{año}^{-1}\right)$ y la tasa de capacidad de transporte de la escorrentía $\left(T C, \mathrm{Mg} \mathrm{ha}^{-1}\right.$ año $\left.^{-1}\right)$ :

$$
E=\text { Pérdida Suelo Anual }=\min \{(F+H) T T C\}
$$

La desagregación del suelo por salpicadura se calcula a partir del volumen de lluvia efectiva $(E R, \mathrm{~mm})$ que es la fracción de la lluvia anual $(R, \mathrm{~mm})$ que alcanza el suelo tras considerar la interceptación de la lluvia por la vegetación $(A, 0-1)$. El volumen de lluvia efectiva se divide en la lluvia que atraviesa la vegetación e impacta directamente sobre la superficie del suelo, conocida como lluvia de trascolación $(D T, \mathrm{~mm})$, y la cantidad de lluvia que es retenida por las ramas y hojas del árbol y que acaba cayendo al suelo por goteo $(L D, \mathrm{~mm})$. El volumen de agua que escurre a través del tronco, escorrentía cortical, se incluye dentro del volumen de agua que cae al suelo desde las hojas y las ramas.

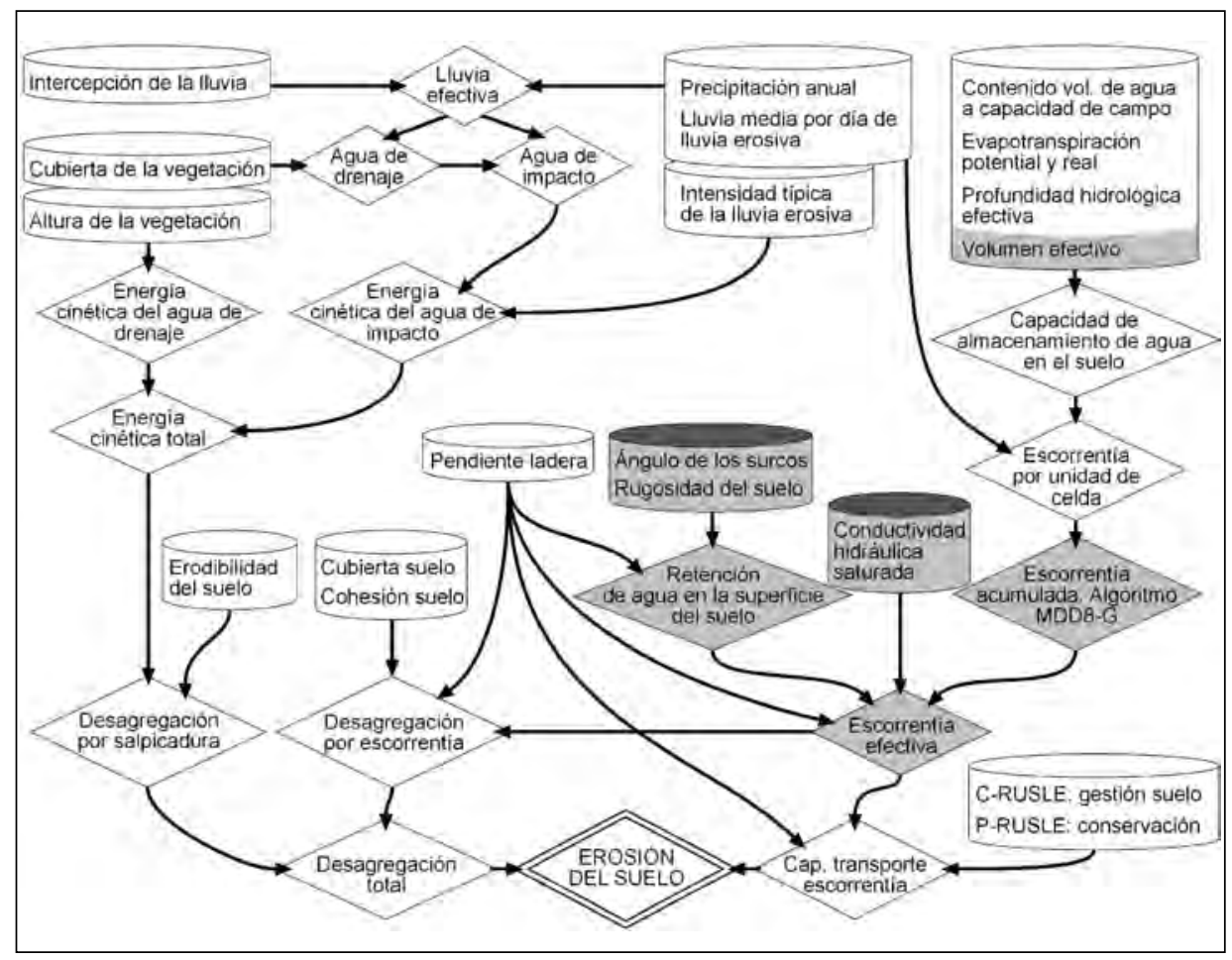

Figura 4. Organigrama del modelo RMMF corregido (los objetos en fondo gris corresponden a las modificaciones introducidas sobre el modelo original) basado en López-Vicente et al. (2008a) 


$$
\begin{gathered}
E R=R A \\
L D=E R C C \\
D T=E R-L D
\end{gathered}
$$

Donde: $C C(\%)$ es el porcentaje de suelo cubierto por la vegetación. La energía efectiva de la lluvia de impacto directo $\left(E(D T), \mathrm{J} \mathrm{m}^{-2}\right)$ se calcula a partir de $D T$ y de la energía cinética de la lluvia $\left(K E, \mathrm{~J} \mathrm{~m}^{-2} \mathrm{~mm}^{-1}\right)$. De entre las numerosas ecuaciones propuestas en las últimas décadas para el cálculo de la energía cinética en función de la intensidad de la precipitación $\left(I, \mathrm{~mm} \mathrm{~h}^{-1}\right)$ se ha seleccionado en este trabajo la ecuación propuesta por Coutinho y Tomas (1995) para áreas de ambiente mediterráneo:

$$
\begin{gathered}
E(D T)=D T K E \\
K E=35.9[1-0.559 \exp (-0.034 /)]
\end{gathered}
$$

La energía efectiva del agua de drenaje $\left(E(L D), \mathrm{J} \mathrm{m}^{-2}\right)$ se calcula en función de la altura media del dosel de la vegetación $(P H, \mathrm{~m})$. En aquellos casos en los que $E(L D)$ es negativo, se asume un valor de cero.

$$
E(L D)=\left(15.8 P H^{0.5}\right)-5.87
$$

La energía efectiva total de la lluvia $\left(E E, \mathrm{~J} \mathrm{~m}^{-2}\right)$ se obtiene a partir de la suma de la energía de la lluvia que impacta directamente sobre la superficie del suelo y de la energía del agua que cae desde la vegetación. La desagregación de partículas del suelo por salpicadura se estima a partir de la energía efectiva total de la lluvia y del índice de erodibilidad del suelo $\left(K, \mathrm{~g} \mathrm{~J}^{-1}\right)$, que es el peso del suelo desagregado de la masa inicial por unidad de energía de la lluvia:

$$
\begin{gathered}
E E=E(D T)+E(L D) \\
F=K E E 10^{-2}
\end{gathered}
$$

La desagregación del suelo debida a la escorrentía se estima en función del volumen de la escorrentía efectiva $(D Q, \mathrm{~mm})$, de la resistencia del suelo a ser desagregado $\left(Z, \mathrm{kPa}^{-1}\right)$, del ángulo de inclinación de la pendiente de la ladera $(S$, radianes) y del porcentaje del suelo cubierto $(G C, \%)$ :

$$
\begin{gathered}
H=Z D Q^{1.5} \sin (S)(1-G C) 10^{-2} \\
Z=\frac{1}{0.5 C O H}
\end{gathered}
$$

Donde: $\mathrm{COH}(\mathrm{kPa})$ es la cohesión de la superficie del suelo en condiciones de saturación.

La lluvia se convierte en escorrentía una vez que el suelo alcanza el punto de saturación y se excede la capacidad de almacenamiento de agua en la superficie. La esco- 
rrentía efectiva $(D Q, \mathrm{~mm})$ se obtiene a partir de la escorrentía acumulada potencial $\left(D Q_{0}, \mathrm{~mm}\right)$, de la conductividad hidráulica saturada para los diferentes tipos de suelo $\left(K_{f s}, \mathrm{~mm} \mathrm{día}^{-1}\right)$, de la máxima capacidad de almacenamiento de agua en la superficie del suelo $\left(S S_{\max }, \mathrm{mm}\right)$ y de la pendiente de la ladera ( $S$, radianes):

$$
\begin{gathered}
D Q=\left(D Q_{0}-K_{/ S}-S S_{\text {mix }}\right) \sin S \\
S S_{\max }=0.5 R G \frac{\sin ^{2}(S I G-S)}{\sin (S I G)} \frac{\cot (S I G+S)+\cot (S I G-S)}{2 \cos (S I G) \cos (S)}
\end{gathered}
$$

Donde: $R G(\mathrm{~mm})$ es la rugosidad de la superficie del suelo, $S$ (radianes) es la pendiente de la ladera y $S I G$ (radianes) es el ángulo de inclinación de los surcos y huellas de la superficie del suelo. El parámetro $S S_{\text {max }}$ se ha calculado según la propuesta de Driessen (1986) y al parámetro $S I G$ se le ha asignado un valor constante de $30^{\circ}$ ( 0.52 radianes) en base al trabajo de Terzoudi et al. (2007). La rugosidad de la superficie del suelo viene dada por la disposición aleatoria u orientada de los fragmentos y agregados del suelo.

Debido a las limitaciones del modelo RMMF corregido para estimar la erosión en las zonas con cursos de agua, se ha establecido un valor máximo de escorrentía, asociado al punto promedio de inicio de las cárcavas, que representa el inicio de los cursos de agua efímeros, de tal modo que se establece un valor máximo de desagregación del suelo y de capacidad de transporte por escorrentía. Este valor umbral se ha utilizado para establecer el cambio entre el flujo acumulado de tipo múltiple (MD) y el de tipo sencillo o concentrado (D8) del algoritmo MDD8-G utilizado para estimar la escorrentía acumulada potencial $\left(D Q_{0}\right)$. La validez de este criterio fue demostrada por López-Vicente y Navas (2008) tras aplicar diferentes algoritmos para la modelización de la escorrentía superficial y la erosión del suelo en una cuenca de características similares y con presencia de cárcavas. El algoritmo de flujo combinado evita los errores de los algoritmos sencillo y múltiple al generar un patrón de flujo más realista mediante la división del flujo en la zona de cabecera entre las celdas situadas aguas abajo y ponderar las diferencias de pendiente y concentrar la escorrentía superficial en un único curso de agua en la zona media de la ladera. La modificación de los algoritmos de flujo combinado con datos reales de campo permite discriminar la erosión laminar característica de las zonas entre-regueros y de baja pendiente de la erosión linear propia de regueros y cárcavas (Chaplot et al., 2005).

El volumen de escorrentía potencial por unidad de celda $\left(Q_{i}, \mathrm{~mm}\right)$ viene definido como una función de la precipitación anual $(R, \mathrm{~mm})$, del valor crítico de almacenamiento de agua en el suelo $\left(R_{c}, \mathrm{~mm}\right)$ y de la lluvia media por día de lluvia erosiva en el año $\left(R_{0}, \mathrm{~mm}\right)$ :

$$
\begin{gathered}
Q_{i}=R \exp \left(\frac{-R_{C}}{R_{0}}\right) \\
R_{C}=1000 \mathrm{MS} B D \mathrm{Vol}_{\mathrm{e} \pi} E H D\left(\frac{E T_{n}}{E T_{\eta}}\right)^{0 . \bar{j}}
\end{gathered}
$$


Donde: $M S$ (\% en peso) es el contenido en peso de agua en el suelo a capacidad de campo (33 $\mathrm{kPa}$ de presión), $B D\left(\mathrm{Mg} \mathrm{m}^{-3}\right)$ es la densidad aparente del suelo, $\operatorname{Vol}_{\text {eff }}(-)$ es el volumen efectivo del suelo, $E H D(\mathrm{~m})$ es la profundidad hidrológica efectiva del suelo y $E T_{a} / E T_{0}(-)$ es el cociente entre la evapotranspiración real y potencial por tipo de suelo y cubierta de vegetación. La capacidad de transporte de la escorrentía (TC, Mg ha$\left.{ }^{1} \mathrm{año}^{-1}\right)$ se obtiene del volumen de escorrentía efectiva $(D Q, \mathrm{~mm})$, de la pendiente del terreno ( $S$, radianes) y de los factores $C$ y $P$ de gestión de los cultivos y de medidas de conservación del suelo del modelo RUSLE (Renard et al., 1997):

$$
T C=C P D Q^{2} \sin (S) 10^{-2}
$$

\subsection{Cálculo de los parámetros del modelo}

En una campaña de campo se recogieron 16 muestras de suelo espaciadas regularmente cada 100 metros. Estas muestras se secaron y tamizaron para separar las fracciones mayor y menor de $2 \mathrm{~mm}$ y calcular su pedregosidad ( $G C$ en la Eq. (10)). Sobre cada submuestra se determinaron la densidad aparente ( $B D$ en la Eq. (15)), granulometría y contenido en peso de agua a capacidad de campo ( $M S$ en la Eq. (15)). A partir de los análisis de granulometría y de los valores propuestos por Morgan (2001) para las diferentes texturas del suelo (Tabla 1), se calcularon los parámetros $\mathrm{K}$ y $\mathrm{COH}$ de las Eqs. (9) y (11), respectivamente.

Tabla 1. Valores de erodibilidad $(\mathrm{K})$ y cohesión $(\mathrm{COH})$ del suelo en función del tipo de textura (Morgan, 2001)

\begin{tabular}{|l|c|c|}
\hline Textura & $\mathbf{K}\left(\mathbf{g ~ J} \mathbf{~}^{-1}\right)$ & COH $(\mathbf{k P a})$ \\
\hline Arenosa & 1.2 & 2 \\
Arenosa franca & 0.3 & 2 \\
Franco arenosa & 0.7 & 2 \\
Franca & 0.8 & 3 \\
Limosa & 1.0 & - \\
Franco limosa & 0.9 & 3 \\
Franco arcillo arenosa & 0.1 & 3 \\
Franco arcillosa & 0.7 & 10 \\
Franco arcillo limosa & 0.8 & 9 \\
Arcillo arenosa & 0.3 & - \\
Arcillo limosa & 0.5 & 10 \\
Arcillosa & 0.05 & 12 \\
\hline
\end{tabular}


Los valores de intercepción de la lluvia por la vegetación ( $A$ en Eq. (2)), de cubierta de la vegetación ( $C C$ en Eq. (3)), de altura de las plantas ( $P H$ en Eq. (7)), de profundidad hidrológica efectiva ( $E H D$ en Eq. (15)) y de evapotranspiración potencial y real $\left(E T_{a} / E T_{0}\right.$ en Eq. (15)) corresponden a los propuestos y calculados por uso del suelo y cubierta de vegetación por López-Vicente (2008) para la Cuenca de Estaña. Sobre los mapas de los parámetros $G C$ y $E H D$ se ha aplicado una máscara correspondiente a la zona de la cárcava, de tal modo que se asume un valor nulo de cubierta del suelo y un valor constante de profundidad hidrológica efectiva equivalente al valor de las zonas de suelo desnudo. Este criterio permite reflejar las condiciones especiales de la superficie del suelo en la zona de la cárcava.

Los valores de rugosidad del suelo ( $R G$ en la Eq. (13)) corresponden a los propuestos en el modelo RUSLE (Renard et al., 1997) para zonas forestales con rugosidad aleatoria $(R G=20.3 \mathrm{~mm})$ y zonas de cultivo con rugosidad orientada por arado de vertedera $(R G=48.3 \mathrm{~mm})$ y por subsolador $(R G=17.8 \mathrm{~mm})$, que son las técnicas de cultivo utilizadas en la zona de estudio. Los valores de conductividad hidráulica saturada ( $K_{f s}$ en la Eq. (12)) y de los factores $C$ y $P$ del modelo RUSLE (Eq. (16)) corresponden a los calculados por López-Vicente (2008) para la Cuenca de Estaña.

El mapa de pendientes y el de escorrentía acumulada potencial se han obtenido a partir del modelo digital de elevaciones (MDE) de la zona de estudio corregido por López-Vicente et al. (2009) y del algoritmo de flujo combinado MDD8 diseñado por Schäuble (2003) y disponible en la extensión Sediment Yield Tools 1.03 - Hydrology para ArcView GIS 3.2. Todos los mapas elaborados para los diferentes parámetros de entrada y resultados obtenidos se han generado a una resolución espacial de $5 \times 5 \mathrm{~m}$, equivalente a la del MDE, y mediante las aplicaciones ArcMap 9.0 y ArcScene9.0.

\section{Resultados}

La modelización de los procesos de escorrentía y erosión en la cuenca de la Cárcava La Coloma incluye la digitalización de la cárcava sobre la ortofoto de la zona de estudio y la validación de las líneas de máxima pendiente del MDE y del mapa de flujo acumulado dentro de la cárcava (Fig. 5a, b y c). El perfil topográfico del curso principal de la cárcava muestra zonas de alta pendiente y otras de baja y nula pendiente que corresponden a áreas de acumulación temporal del suelo erosionado aguas arriba y transportado dentro de la cárcava (Fig. 5d). Atendiendo a los usos del suelo y a los parámetros de pendiente y morfología de la red de drenaje, se ha dividido la Cárcava La Coloma en cuatro sectores diferentes (Fig. 5). En los sectores I y II la cárcava se circunscribe a su curso principal mientras que en los sectores III y IV se extiende lateralmente, dando lugar a un sistema de cárcavas dendrítico y de mayor extensión (Tabla 2). 


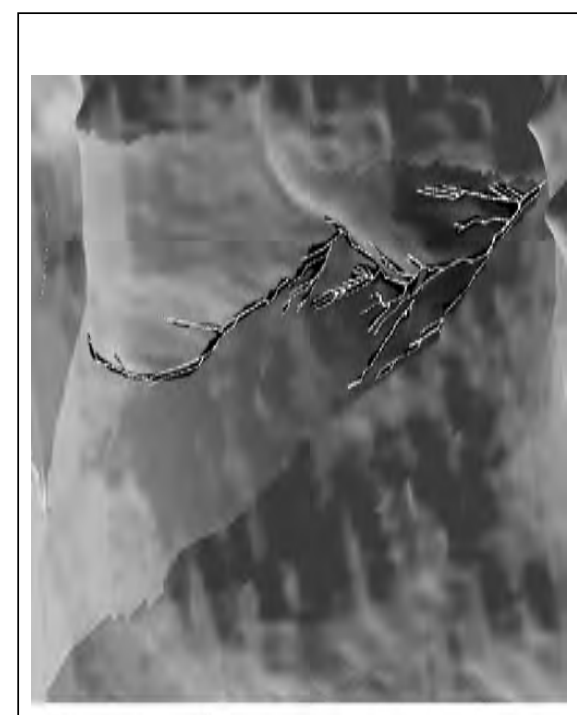

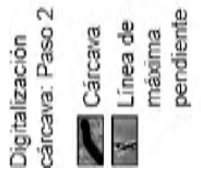

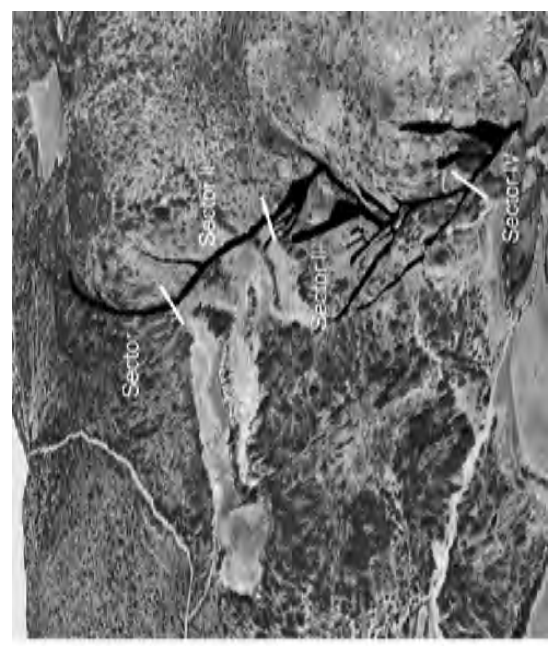

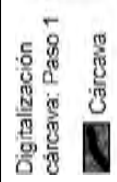
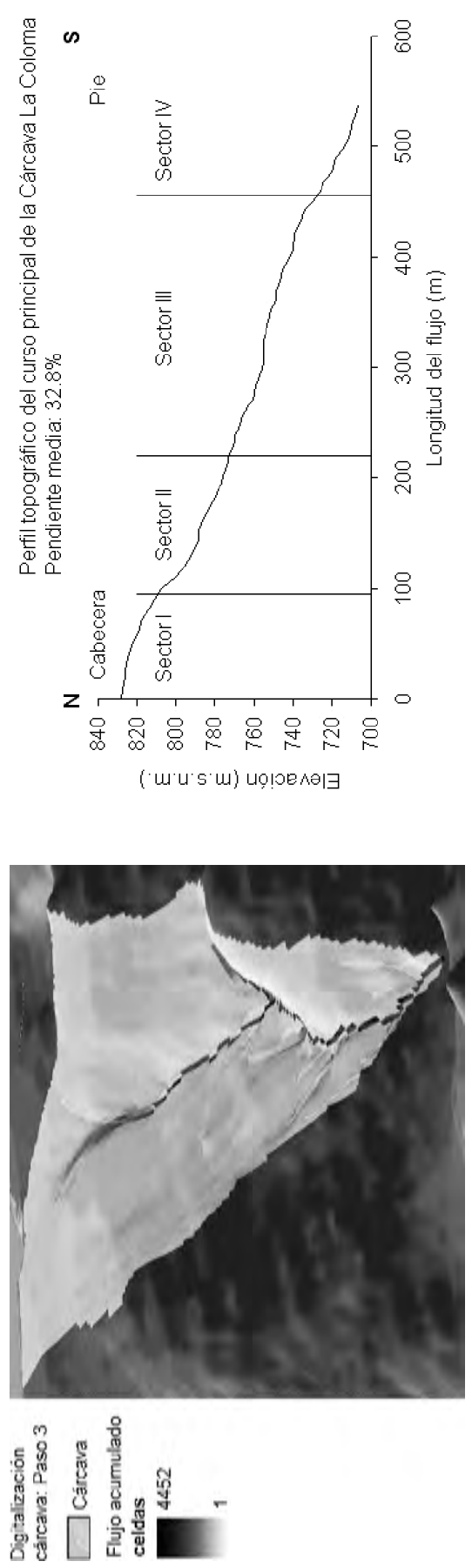

$\stackrel{\frac{1}{2}}{3}$

$\lambda \geqslant$

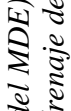

$\frac{1}{2} \div$

$\therefore \frac{2}{8}$

空

马

E

을

离

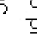

몸

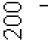

马

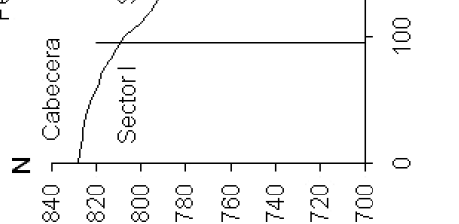

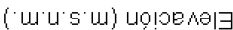

ปี

ปั

홍

¿.

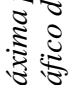

ฐ

$\approx$ :

¿

运

ह 2

$\approx 0$

हैं

20

0 ن

고ำ

존

원

ษ

כ) $: 3$

ะ

ำ

:

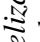

ह

ㅎำ

$\approx$

ป స

ธิ

(2)

离

ป

2.

$\stackrel{\infty}{2}: \frac{1}{3}$

ㄴ

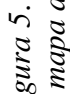




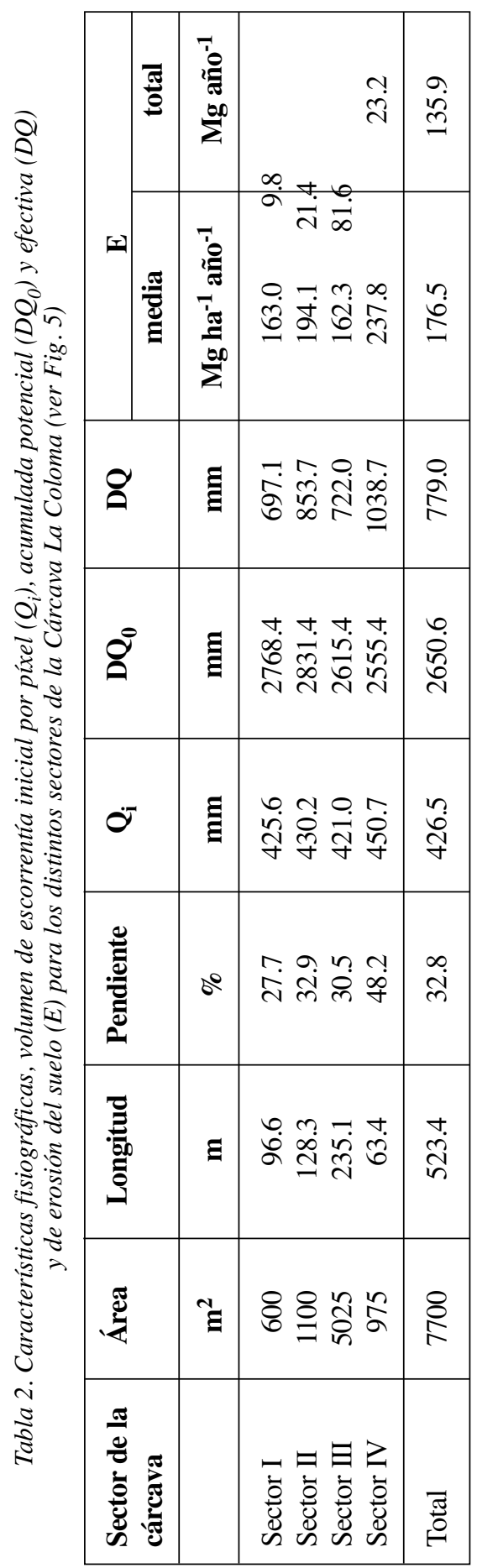




\subsection{Escorrentía y erosión en la cuenca de estudio}

El valor umbral que se ha calculado como límite máximo del volumen de escorrentía es de $3100.8 \mathrm{~mm}$ por píxel y representa la escorrentía acumulada potencial máxima dentro de la cárcava (Fig. 6a). La proximidad de la cabecera de la cárcava con la divisoria de la cuenca explica el bajo valor del umbral calculado para el cambio del algoritmo de flujo múltiple al sencillo así como el aspecto dendrítico del mapa del parámetro $D Q_{0}$. El mapa de la escorrentía efectiva muestra una distribución compleja del volumen de escorrentía generada, en el que los procesos de infiltración y las diferencias de pendiente explican los valores obtenidos (Fig. 6b). El 0.3\% de la superficie del mapa de escorrentía efectiva tiene un valor nulo y corresponde a zonas de divisoria.

El mapa de desagregación por salpicadura muestra un fuerte control de este proceso por parte de los diferentes usos del suelo (Fig. 7a), mientras que el mapa de desagregación por escorrentía refleja la importancia del proceso de concentración del flujo superficial y de las zonas de elevada pendiente (Fig. 7b). La desagregación por salpicadura representa el $70 \%$ de la desagregación total del suelo estimada mediante el modelo RMMF corregido.

La pérdida de suelo promedio estimada para la Cuenca de la Cárcava La Coloma es de $33.9 \mathrm{Mg} \mathrm{ha}^{-1} \mathrm{año}^{-1}$ (Fig. 8), siendo la capacidad de transporte de la escorrentía el factor limitante de la erosión en el $88 \%$ de su superficie. Esta tasa de erosión está por encima de la erosión promedio estimada por ICONA (1980-90) para el conjunto de España (27 Mg ha-1 $\mathrm{año}^{-1}$ ) durante el periodo 1980-1990. El valor de pérdida de suelo promedio corresponde a una tasa de erosión superior al máximo tolerable y señala la existencia de procesos irreversibles de erosión y degradación del terreno. La zona de las divisorias y de cabecera de las líneas de flujo presentan las menores tasas de erosión. La superficie de la cuenca con una tasa de erosión alta (de 8 a $20 \mathrm{Mg} \mathrm{ha}^{-1} \mathrm{año}^{-1}$ ) y muy alta (de 20 a $40 \mathrm{Mg} \mathrm{ha}^{-1} \mathrm{año}^{-1}$ ) es del $17 \%$ y con erosión irreversible (mayor de $40 \mathrm{Mg} \mathrm{ha}^{-1}$ año $^{-1}$ ) del $23 \%$. El porcentaje de la cuenca con erosión baja (menor de $4 \mathrm{Mg} \mathrm{ha}^{-1}$ año $^{-1}$ ) y tolerable (de 4 a $8 \mathrm{Mg} \mathrm{ha}^{-1}$ año $^{-1}$ ) es del 54 y $6 \%$, respectivamente. La tasa de erosión promedio en el depósito coluvial de ladera $\left(96.9 \mathrm{Mg} \mathrm{ha}^{-1} \mathrm{año}^{-1}\right)$ y sobre materiales arcillosos (67.0 $\mathrm{Mg} \mathrm{ha}^{-1} \mathrm{año}^{-1}$ ) se sitúa por encima de la media para el conjunto de la cuenca, mientras que los suelos desarrollados sobre calizas $\left(17.8 \mathrm{Mg} \mathrm{ha}^{-1} \mathrm{año}^{-1}\right)$ y sobre el depósito de fondo de valle plano (5.5 $\left.\mathrm{Mg} \mathrm{ha}^{-1} \mathrm{año}^{-1}\right)$ son los menos erosionables. La pérdida de suelo estimada por uso del suelo varía notablemente para los diferentes usos, apareciendo las tasas más altas en aquellas cubiertas con poco desarrollo de la vegetación (Tabla 3). 

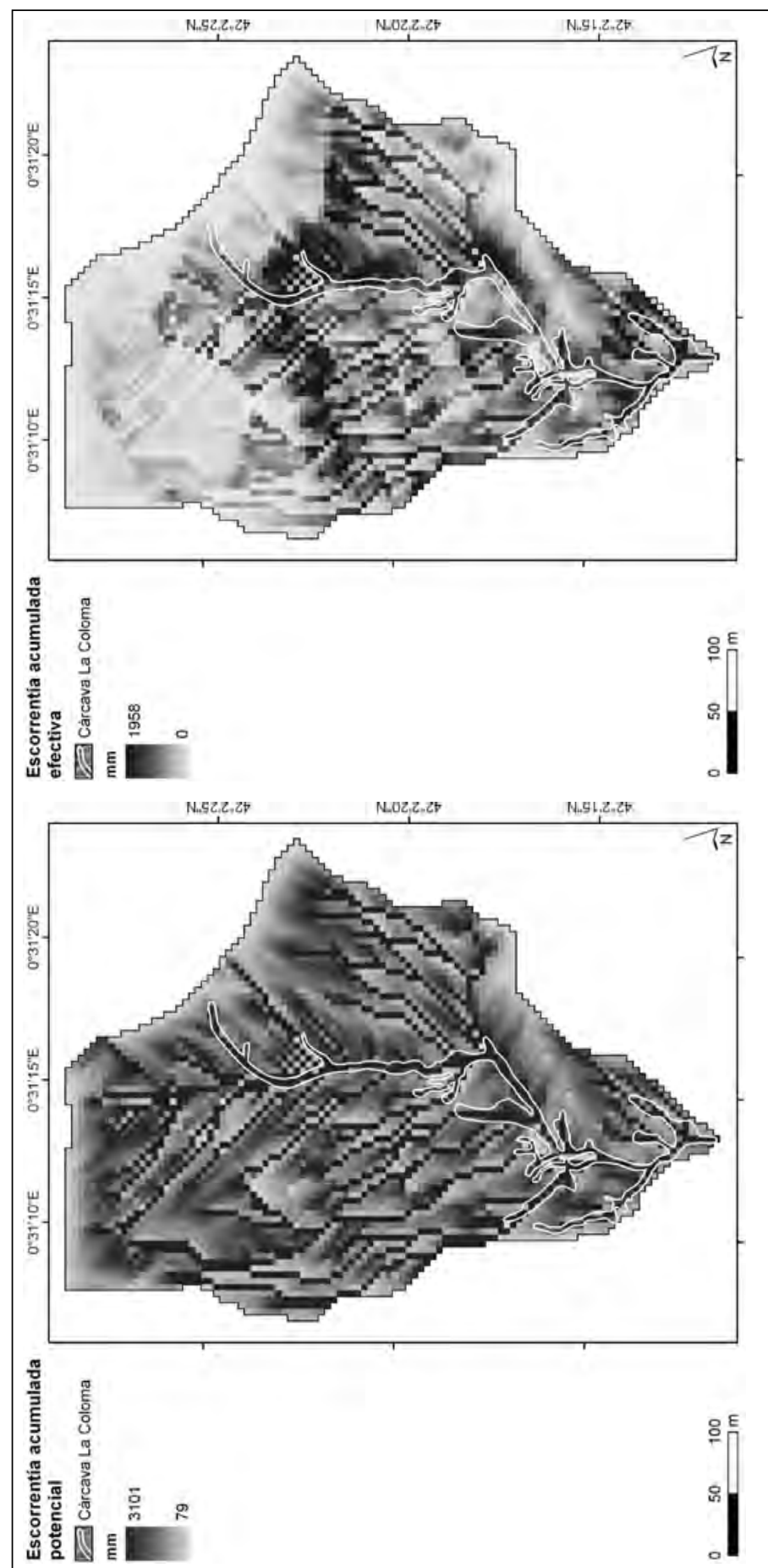

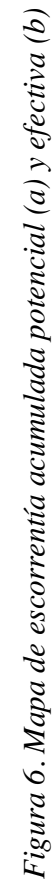




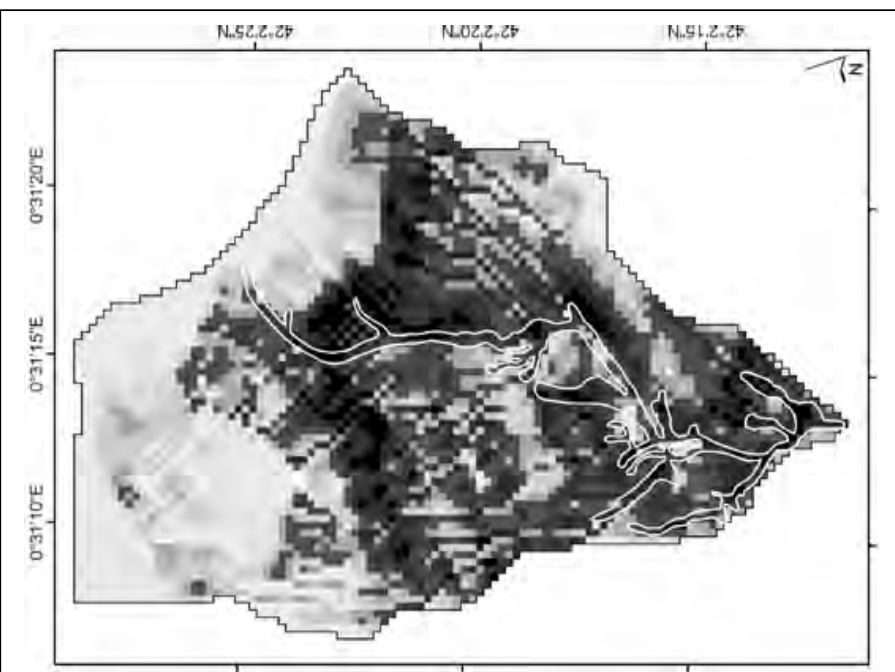

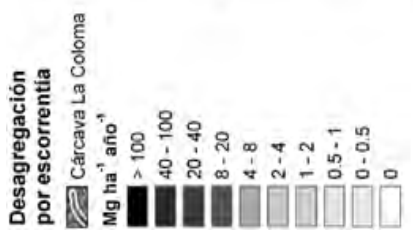
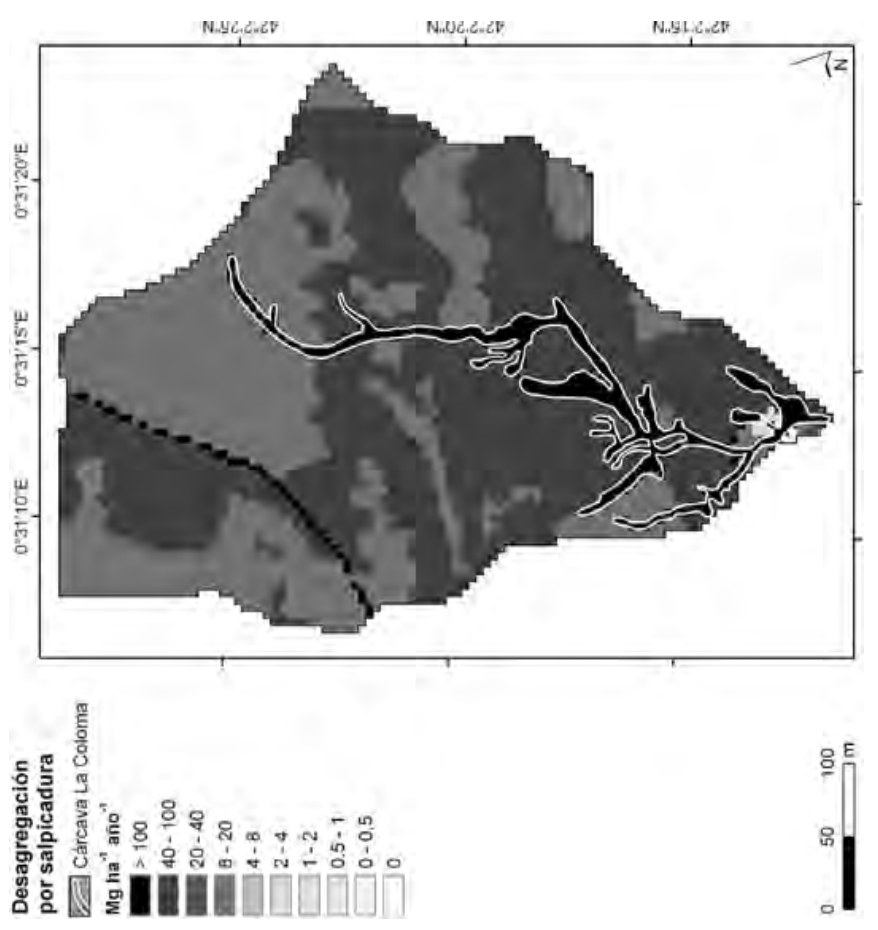

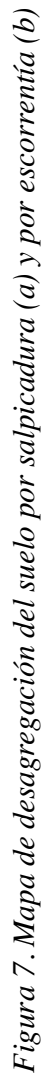




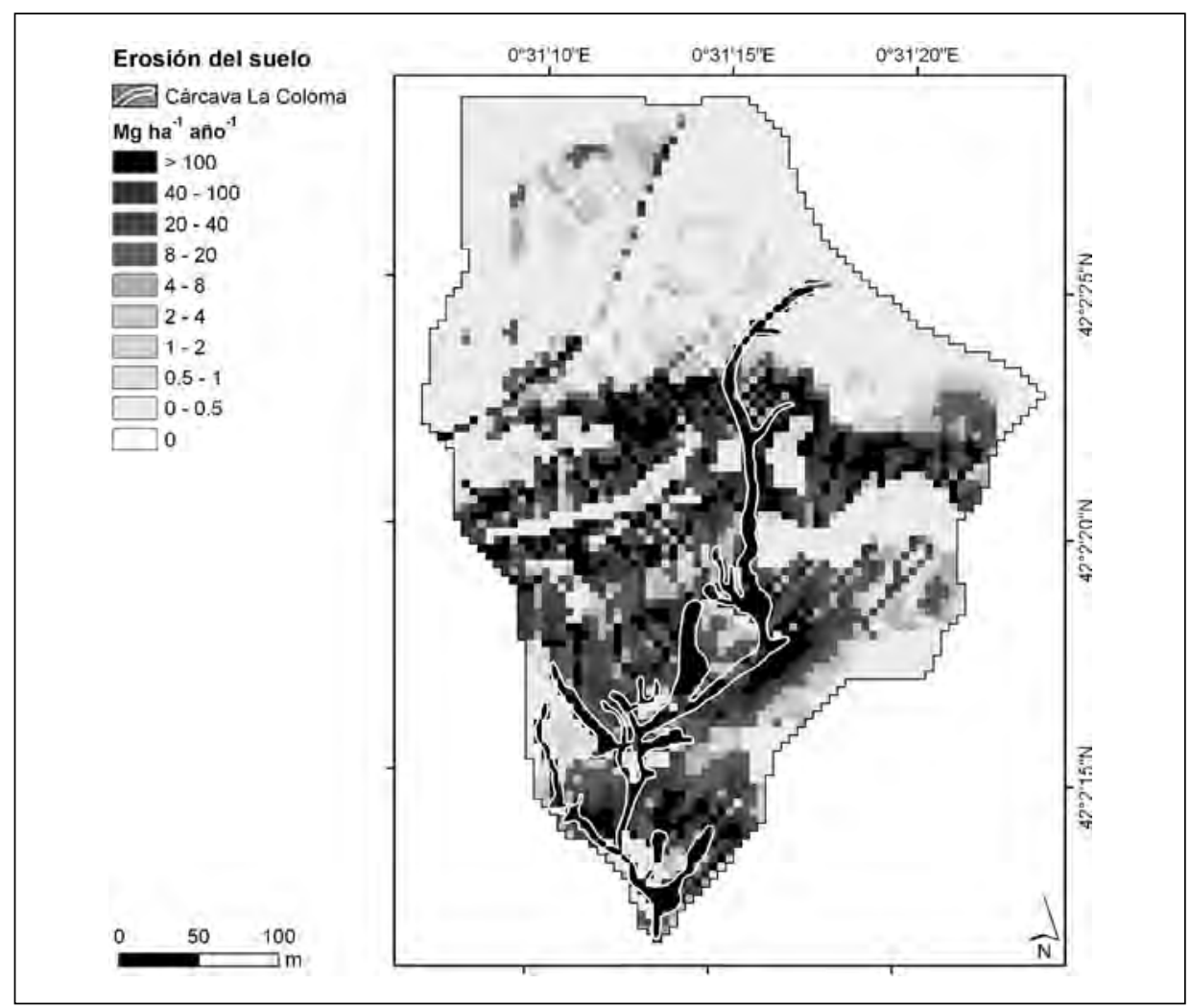

Figura 8. Mapa de erosión del suelo estimada mediante el modelo RMMF corregido en la cuenca de la Cárcava La Coloma (Prepirineo oscense)

Tabla 3. Erosión del suelo anual por uso del suelo estimado mediante el modelo RMMF corregido en la cuenca de la Cárcava La Coloma

\begin{tabular}{|l|c|}
\hline Uso del suelo & Pérdida de suelo anual $\left(\mathbf{M g ~ h a} \mathbf{~ a n ̃ o}^{\mathbf{- 1}}\right)$ \\
\hline Camino & 65.9 \\
Cebada & 56.9 \\
Pasto & 1.5 \\
Campo abandonado antiguo & 1.0 \\
Bosque mediterráneo denso & 0.5 \\
Bosque mediterráneo abierto & 37.0 \\
Arbusto denso & 0.9 \\
Arbusto disperso & 51.2 \\
Chopo & 0.7 \\
\hline
\end{tabular}




\subsection{Escorrentía y erosión en la Cárcava La Coloma}

La escorrentía inicial por unidad de celda, acumulada potencial y efectiva muestra valores muy parecidos en los cuatro sectores en los que se divide la cárcava, debido a la homogeneidad fisiográfica de los distintos sectores (Tabla 2). El volumen de escorrentía efectiva anual exportado a través de la cárcava es de $779 \mathrm{~mm}$ año ${ }^{-1}$ y la erosión promedio en el conjunto de la cárcava es de $176.5 \mathrm{Mg} \mathrm{ha}^{-1} \mathrm{año}^{-1}$, alcanzando un valor máximo de $502.6 \mathrm{Mg} \mathrm{ha}^{-1}$ año $^{-1}$. Las menores tasas de erosión se han obtenido en los sectores I y III, situados en la cabecera de la cárcava el primero, y en la zona de mayor extensión lateral el segundo. Estos sectores presentan los menores valores de escorrentía efectiva y de pendiente. El sector IV de la cárcava, situado a los pies de la ladera, presenta la mayor tasa de erosión, con un valor promedio de $237.8 \mathrm{Mg} \mathrm{ha}^{-1} \mathrm{año}^{-1}$. Estos valores son superiores a los obtenidos por Casalí et al. (1999) en cárcavas desarrolladas sobre margas miocenas en Navarra (erosión media de $26.6 \mathrm{Mg} \mathrm{ha}^{-1} \mathrm{año}^{-1}$ ) y a los obtenidos por Desir y Marín (2007) en arcillas y margas de las Bardenas Reales (erosión media de 24.6 $\pm 13 \mathrm{Mg} \mathrm{ha}^{-1}$ año $^{-1}$ ) y similares al rango de valores obtenidos por Benito et al. (1992) en badlands de Lupiñén, al sur de las Sierras Exteriores (tasas entre 32 y $400 \mathrm{Mg} \mathrm{ha}^{-1}$ mediante agujas de erosión y entre 24 y $485 \mathrm{Mg} \mathrm{ha}^{-1}$ con perfiles microtopográficos para un periodo de observación de entre 7 y 15 meses, respectivamente y en ambos casos). La menor precipitación anual registrada en el área de estudio de Casalí et al. (1999) y de Desir y Marín (2007) explicarían las diferentes tasas obtenidas por estos autores.

Las limitaciones del modelo RMMF corregido y los resultados obtenidos sugieren la necesidad de incluir en futuras investigaciones un módulo de erosión y transporte del sedimento específico para zonas con cursos de agua. La calibración de este nuevo módulo mediante hidrogramas y sedigramas permitirá desarrollar un nuevo algoritmo para optimizar las predicciones del volumen de escorrentía efectiva generada.

Estos resultados ponen de manifiesto la utilidad de las técnicas SIG en la aplicación de modelos de erosión para el estudio de la erosión y su representación espacial, tal y como Navas et al. (2005) realizaron en zonas mediterráneas y Pirenaicas a escala de cuenca.

\section{Conclusiones}

La aplicación del modelo RMMF corregido mediante el algoritmo MDD8-G de flujo combinado y asociado a las cárcavas permite la representación precisa de la escorrentía acumulada potencial espacialmente distribuida. El mapa de escorrentía efectiva pone de manifiesto la importancia de los procesos de infiltración, de acumulación superficial del agua y de pendiente del terreno en el volumen final de escorrentía superficial generada, que va a conducir los procesos de erosión y transporte del suelo.

Las tasas de desagregación por salpicadura dependen en gran medida de los diferentes usos del suelo, mientras que el mapa de desagregación por escorrentía refleja la importancia del proceso de concentración del flujo superficial y de las zonas de elevada 
pendiente. La desagregación por salpicadura representa más de la mitad de la desagregación total del suelo estimada mediante el modelo RMMF corregido. La capacidad de transporte de la escorrentía es el factor limitante de la erosión en la casi totalidad de la zona de estudio. El volumen de escorrentía efectiva total anual es de $779 \mathrm{~mm}$ año ${ }^{-1}$ dando lugar a una tasa de erosión para la Cárcava La Coloma que quintuplica la erosión promedio estimada para el conjunto de la cuenca. La pérdida de suelo estimada en los diferentes sectores de la cárcava es similar o superior a la medida en campo en zonas del Prepirineo de similares condiciones climáticas. Los cultivos, caminos y las áreas de elevada pendiente son las principales áreas contributivas del suelo erosionado al canal principal de la cárcava.

La aplicación del modelo RMMF corregido resulta de interés y puede ser aplicado a todos aquellos ambientes en los que aparecen y se desarrollan las cárcavas, que son características y frecuentes en áreas degradadas o bajo una intensa dinámica erosiva. Sin embargo, las limitaciones del modelo RMMF corregido para representar procesos de erosión y transporte del sedimento en canales y cárcavas permanentes da lugar a la subestimación del volumen de escorrentía generada y de la cantidad de suelo erosionado y exportado en la cárcava. Por lo tanto, resulta necesaria la inclusión de nuevos factores relativos a la dinámica erosiva en canales, que además consideren el efecto del incremento de la concentración de partículas en suspensión y disolución en el flujo que circula dentro de la cárcava.

\section{Agradecimientos}

Este trabajo de investigación ha sido financiado a través de los proyectos de la CICYT: RADIERO (REN2002-02702/GLO) y REM (CGL2005-02009/BTE).

\section{Referencias bibliográficas}

Benito, G., GutiÉRrez, M., SAncho, C. (1992). Erosion Rates in Badland Areas of the Central Ebro Basin (NE-Spain). Catena, 19: 269-286.

Bou Kheir, R., Chorowicz, J., Abdallah, C., Dhont, D. (2008). Soil and bedrock distribution estimated from gully form and frequency: A GIS-based decision-tree model for Lebanon. Geomorphology, 93(4/3): 482-492.

CASAlí, J., LóPez, J.J., GiRÁldez, J.V. (1999). Ephemeral gully erosion in southern Navarra (Spain). Catena, 36: 65-84.

Castillo, V.M., Mosch, W.M., García, C.C., BARberá, G.G., CANO, J.A.N., LÓPEZBERMÚDEZ, F. (2007). Effectiveness and geomorphological impacts of check dams 
for soil erosion control in a semiarid Mediterranean catchment: El Cárcavo (Murcia, Spain). Catena, 70(3): 416-427.

Chaplot, V., Giboire, G., Marchand, P., Valentin, C. (2005). Dynamic modelling for linear erosion initiation and development under climate and land-use changes in northern Laos. Catena, 63: 318-328.

CoutinHo, M.A., ToMÁs, P.P. (1995). Characterization of raindrop size distributions at the Vale Formoso Experimental Erosion Center. Catena, 25: 187-197.

De Santisteban, L.M., Casalí, J., LóPeZ, J.J. (2006). Assessing soil erosion rates in cultivated areas of Navarre (Spain). Earth Surf. Process. Landforms, 31: 487-506.

de Vente, J., Poesen, J., Verstraeten, G., Van Rompaey, A., Govers, G. (2008). Spatially distributed modelling of soil erosion and sediment yield at regional scales in Spain. Global and Planetary Change, 60(3-4): 393-415.

DESIR, G., MARÍN, C. (2007). Factors controlling the erosion rates in a semi-arid zone (Bardenas Reales, NE Spain). Catena, 71(1): 31-40.

DRIESSEN, P.M. (1986). The water balance of soil. En: Modeling of Agricultural Production: Weather, Soils and Crops (van Keulen, H., Wolf, J., Eds.). pp. 76-116, Pudoc, Wageningen, The Netherlands.

FAulKner, H., AleXander, R., TeEuw, R., Zukowskyj, P. (2004). Variations in soil dispersivity across a gully head displaying shallow sub-surface pipes, and the role of shallow pipes in rill initiation. Earth Surface Processes and Landforms, 29(9): 1143-1160.

FERnÁndeZ, S., MARQuíneZ, J., MENÉNDEZ-DuARTE, R. (2008). A sapping erosion susceptibility model for the southern Cantabrian Range, North Spain. Geomorphology, 95: 145-157.

Gordon, L.M., Bennett, S.J., Bingner, R.L., Theurer, F.D., Alonso, C.V. (2007). Simulating ephemeral gully erosion in AnnAGNPS. Transactions of the ASABE, 50(3): 857-866.

ICONA (1980-1990). Mapas de Estados Erosivos. Cuencas Hidrográficas del Ebro, Guadiana, Guadalquivir, Júcar, Pirineo, Segura, Sur de España, Tajo,... ICONA, Ministerio de Agricultura, Pesca y Alimentación, Madrid.

LÓPEZ-VICENTE, M. (2008). Erosión y redistribución del suelo en agroecosistemas mediterráneos: modelización predictiva mediante SIG y validación con ${ }^{137}$ Cs (Cuenca de Estaña, Pirineo Central). Universidad de Zaragoza, 212 pág., Zaragoza. ISBN: 978-84-7733-008-0.

LóPez-Vicente, M., NAVAs, A., Machín, J., Gaspar, L. (2006). Modelización de la pérdida de suelo en una cuenca endorreica del Pirineo oscense. Cuadernos de Investigación Geográfica, 32: 29-42. 
LÓPEZ-ViCENTE, M., NAVAS, A. (2008). Routing soil particles in a distributed model with GIS in Mediterranean agricultural landscapes and implications for wetland conservation. Geophysical Research Abstracts, 10: 11705.

LóPeZ-Vicente, M., NAVAS, A., Machín, J. (2008a). Modelling soil detachment rates in rainfed agrosystems in the south-central Pyrenees. Agricultural Water Management, 95(9): 1079-1089.

LóPEZ-Vicente, M., NAVAS, A., MAChÍN, J. (2008b). Identifying erosive periods by using RUSLE factors in mountain fields of the Central Spanish Pyrenees. Hydrology and Earth System Science, 12(2): 523-535.

LóPeZ-Vicente, M., NAVAs, A., Machín, J. (2009). Geomorphic mapping in endorheic catchments in the Spanish Pyrenees: an integrated GIS analysis of karstic features. Geomorphology: DOI 10.1016/j.geomorph.2008.03.014

Martínez-CASASnOvas, J.A., Antón-Fernández, C., RAMOs, M.C. (2003). Sediment production in large gullies of the Mediterranean area (NE Spain) from high-resolution digital elevation models and geographical information systems analysis. Earth Surf. Process. Landforms, 28: 443-456.

MARZOLFF, I., RIES, J.B. (2007). Gully erosion monitoring in semi-arid landscapes. Zeitschrift fur Geomorphologie, 51(4): 405-425.

MEnÉndeZ-DuARTE, R., MARQuínEZ, J., FERnÁndEZ-MENÉNDEZ, S., SANTOS, R. (2007). Incised channels and gully erosion in Northern Iberian Peninsula: Controls and geomorphic setting. Catena, 71(2): 267-278.

Morgan, R.P.C. (2001). A simple approach to soil loss prediction: a revised MorganMorgan-Finney model. Catena, 44: 305-322.

Morgan, R.P.C., Morgan, D.D.V., Finney, H.J. (1984). A predictive model for the assessment of soil erosion risk. Journal of Agricultural Engineering Research, 30: 245-253.

NaVAs, A., Machín, J., Soto, J. (2005). Assessing soil erosion in a Pyrenean mountain catchment using GIS and fallout ${ }^{137}$ Cs. Agriculture, Ecosystems \& Environment, 105: 493-506.

Nadal-Romero, E., Latron, J., Martí-Bono, C., Regués, D. (2008). Temporal distribution of suspended sediment transport in a humid Mediterranean badland area: The Araguás catchment, Central Pyrenees. Geomorphology, 97(3-4): 601-616.

Renard, K.G., Foster, G.R., Weesies, G.A., McCoOl, D.K., Yoder, D.C. (1997). Predicting Soil Erosion by Water: A Guide to Conservation Planning with the Revised Universal Soil Loss Equation (RUSLE). Handbook \#703. US Department of Agriculture, Washington, DC. 
RIES, J.B., MARZOLFF, I. (2003). Monitoring of gully erosion in the Central Ebro Basin by large-scale aerial photography taken from a remotely controlled blimp. Catena, 50(2-4): 309-328.

SCHÄUBLE, H. (2003). HydroTools 1.0 for ArcView 3.x. En: TERRACS - Geographical

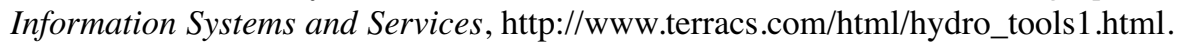

Terzoudi, C.B., Gemtos, T.A., Danalatos, N.G., Argyrokastritis, I. (2007). Applicability of an empirical runoff estimation method in central Greece. Soil \& Tillage Research, 92: 198-212.

Vigiak, O., Sterk, G., Romanowicz, R.J., Beven, K.J. (2006). A semi-empirical model to assess uncertainty of spatial patterns of erosion. Catena, 66: 198-210. 